Journal of Computer Science 4 (10): 784-791, 2008

ISSN 1549-3636

(C) 2008 Science Publications

\title{
Local Beam Search Algorithm for Power Saving in Clustered Ad-Hoc Networks
}

\author{
${ }^{1}$ Arwa Zabian and ${ }^{2}$ Fadi Al-Kalani \\ ${ }^{1}$ Department of CS, Jadara University, Irbid, Jordan \\ ${ }^{2}$ Department of Computer Science, Irbid National University, Irbid, Jordan
}

\begin{abstract}
An ad-Hoc network is an infrastructures network consisting of mobile moving nodes. Every node in network performs as a router or a package forwarder. Energy consumption of network interfaces can be significant. There is a relationship between the transmission power and the distance traveled by a packet, increasing the transmission power increases the reachable area at the cost of high power consumption. Reducing the transmission power reduces the number of nodes reached but consumes less energy. We propose the use of searching algorithms (local beam search) to organize the nodes of a single cluster in a tree, in a manner that all the nodes are distributed in levels given a determined distance to each other. All the communication was done by levels. Substituting in that long distance communication by a multihop communication. Reducing in the power consumption for each communication. Results showed that power remaining at the destination node inversely related to the distance between the two communicating nodes. Power increased at the destination node if the distance between the two communicating nodes decreased.
\end{abstract}

Key words: Searching algorithms, power consumption, multihop communication, local beam search algorithm

\section{INTRODUCTION}

Multihop communication is one of the main enablers in reducing power consumption in ad hoc networks. The energy required for communication between two arbitrary nodes A and B is strongly dependent on the distance between the two nodes. $\mathrm{E}=\mathrm{B} \cdot \mathrm{d}^{\mathrm{y}}$ where $\mathrm{y}>1$ is the path loss exponent depending on the RF environment and $\mathrm{B}$ is a proportionality constant describing the overhead per bit. Given this super linear relationship between energy and distance, generally using several short intermediate hops to send a bit is more energy efficient than using one longer hop.

However, the use of infinite number of hops over the smallest possible distances is impractical for two reasons:

- The number of intermediate hops is limited by the number of nodes between $\mathrm{A}$ and $\mathrm{B}$

- The energy is not limited to the energy radiated through the antenna. There is also the energy dissipated in the radio for receiving a bit and reading a bit for retransmission

However, the major part of energy consumption in a node is done in the communication taking in consideration that the power consumed in the computation can be minimized. For that, a good power saving algorithm must minimize the amount of communication. Power saving is the most relevant metrics in wireless networks. Experimental measurements indicate that communication cost in wireless Ad-hoc networks is at least two orders of magnitude higher than computation cost in term of consumed power.

Notice that, the coverage problem and location identification are two problems strictly related to the power saving problem.

In this research, we propose a power saving mechanism for clustered ad-hoc networks. Where, it is used the local beam search ${ }^{[7]}$, in order to organize the nodes of the cluster in a tree rooted by the head cluster, in a manner that the communication between any two nodes in the cluster (either neighbors) is done by levels (multihops). Our system is applied on that proposed in $^{[6]}$, in which given a set of mobile nodes, the nodes are organized in cluster given the transmission range to obtain better network performance.

In each cluster, the head cluster (leader) has the role to coordinate the communication between the nodes of the cluster. However, the nodes of the single cluster are in the transmission range of the leader can communicate to it directly or by other nodes. 
Our idea is to distribute the power consumption between the nodes of the cluster in order to increase the cluster life time. In wireless networks, increasing the transmission range allows the reaching of more nodes but requires high power consumption. For that, we substitute the high distance communication in the cluster by multihops communication that reduces the power needed at each intermediate node for the communication and to distribute the power consumption between these nodes and to reduce the signal dissipation.

\section{MATERIALS AND METHODS}

Algorithm description: The system as any network model is represented by a graph $\mathrm{G}=(\mathrm{V}, \mathrm{E})$ where $\mathrm{V}$ is a set of mobile nodes, $|\mathrm{V}|=\mathrm{n}$ and $\mathrm{E}$ is a set of bidirectional links.

We will organize the nodes of each cluster in a dynamic tree rooted by the head cluster, in a manner that, the nodes that are far from the head cluster, but are in its transmission range, can communicate with it by a way of other nodes close to it, reducing in that, the power consumption in sending packets between any two nodes in the same cluster and increasing the cluster life time.

Our algorithm works in phases, where in the first phase the neighbors of the root are defined and then, in the successive phases in the same manner each node defines the closest nodes to it. The number of phases must be equal to the height of the tree that can be at most 3 (because if the packet travels more than 3 hops the signal dissipation will increase). For that, we limit the number of child to each node to $\mathrm{k}$ where $2 \leq \mathrm{k} \leq 5$ insuring that the tree does not become separated due to nodes mobility.

Definitions: we will explain some definitions that are used in the algorithm description:

- $\mathrm{K}$ represents the number of to each node $2 \leq \mathrm{k} \leq 5$

- $\mathrm{T}_{\mathrm{r}}$ represents the response time, it is calculated as the time from which the leader sends the request to the time in which it receives a response, ignoring the attenuation of the signal for small distance. $T_{r}=2 d / v$ where $d$ is the distance between the two communicating nodes and $\mathrm{v}$ is the propagation speed that is considered equal for all the nodes. $T_{r}$ $=\mathrm{T}_{\mathrm{A}}-\mathrm{T}_{\mathrm{S}}$.

- $\mathrm{T}_{\mathrm{A}}$ is the arriving time of the response message

- $\mathrm{T}_{\mathrm{S}}$ is the time at which the leader has sent a message

- $\mathrm{r}$ is the transmission range
- $\mathrm{f}$ is a fitness function by which the nodes are selected by levels: $f=r * 20 / 100$ that represents the selected distance between the two nodes.

- $\quad P$ is the power level and it takes values from 1 to $j$ where the leader must have power equal to $\mathrm{j}$, given the algorithm proposed $\mathrm{in}^{[6]}$.

\section{Revised Dynamic Search Tree Algorithm (RDSTA):} RDSTA is organized to be designed with two phases.

Setting phase: In this phase, the leader selects the nodes of its cluster based on their distance to it. From the cluster formation phase in DHCEA (Dynamic Head Cluster Election Algorithm $)^{[6]}$, it is assumed that a large subset of the nodes of the cluster is in the transmission range of the leader but at different distances.

- The leader broadcasts a Distance Setting Message (DSM) and set a time out $\mathrm{T}_{\mathrm{r}}$

- All the nodes that hear the hello message and want to join to the leader must send a response message that contains its identity and its power level

- The response messages are inserted in a queue based on the arriving time $\mathrm{T}_{\mathrm{A}}$

- The leader selects the received responses one by one DOIng the following comparisons

- If $T_{r} \leq 2 f / v$ and $j / 2 \leq p \leq j$, the corresponding node is close to the root and has enough power needed to carry out the communication between the root and the other nodes in the tree and will be denoted as discovered. Otherwise, the visited node will be deleted from the queue because it is far from the root or because its power is not enough to connect the leader with the rest of the tree

- Step 4 is repeated until satisfying the condition $2 \leq \mathrm{k} \leq 5$

- All the nodes with $\mathrm{k}>5$ are deleted from the queue

Tree construction phase: Steps 1-6 will be repeated for each discovered node until all the nodes of the cluster are connected to each other and each node knows who its parent is and who their children are.

In this manner, the distance between each node and its child in the tree is fixed and equal to $\mathrm{r} * 20 \%$. That means, the nodes at distance two levels in the tree are distant from the root about $\mathrm{r} * 40 \%$ and so on. The nodes with distance more than $\mathrm{r} * 80 \%$ from the root will be ignored because if the root needs to communicate directly to them, the transmission will be too weak.

The nodes with distance between $\mathrm{r} * 20 \% \leq \mathrm{d} \leq$ $\mathrm{r} * 40 \%$ (between levels ) will be added to the lower 


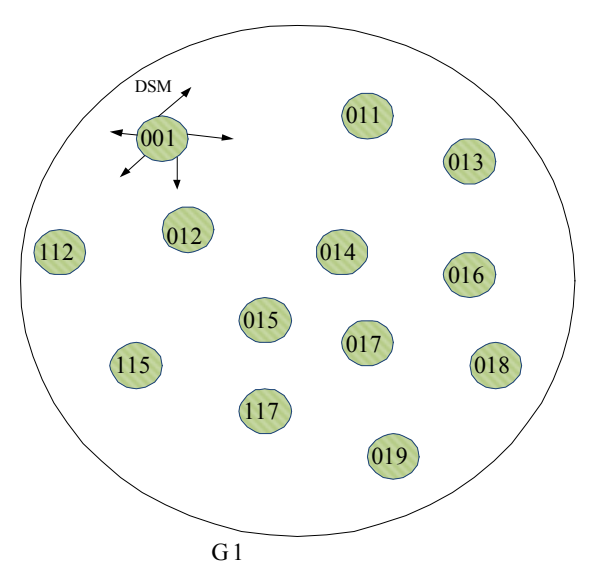

Fig. 1: Ad-hoc nodes cluster distribution example
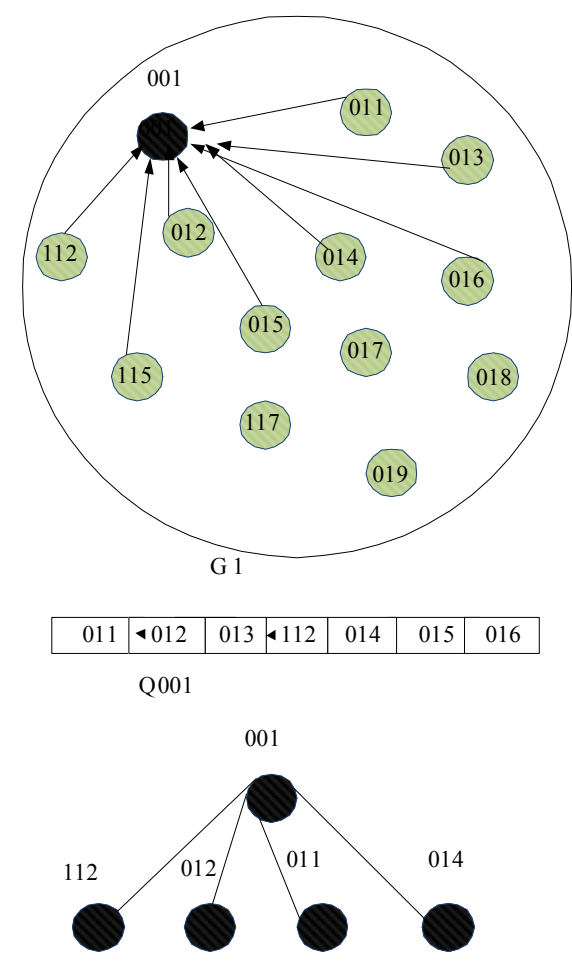

Fig. 2: Setting phase

level, because they can carry a communication with more power staying in the lower level than in the higher level.

Example: Figure1 showed a cluster of Ad-hoc nodes as described in ${ }^{[6]}$. Where 001 is the leader of the cluster. The cluster is composed on 12 nodes. In Fig. 1 the leader starts broadcasting DSM message. Figure 2, represents the setting phase, in which each node has

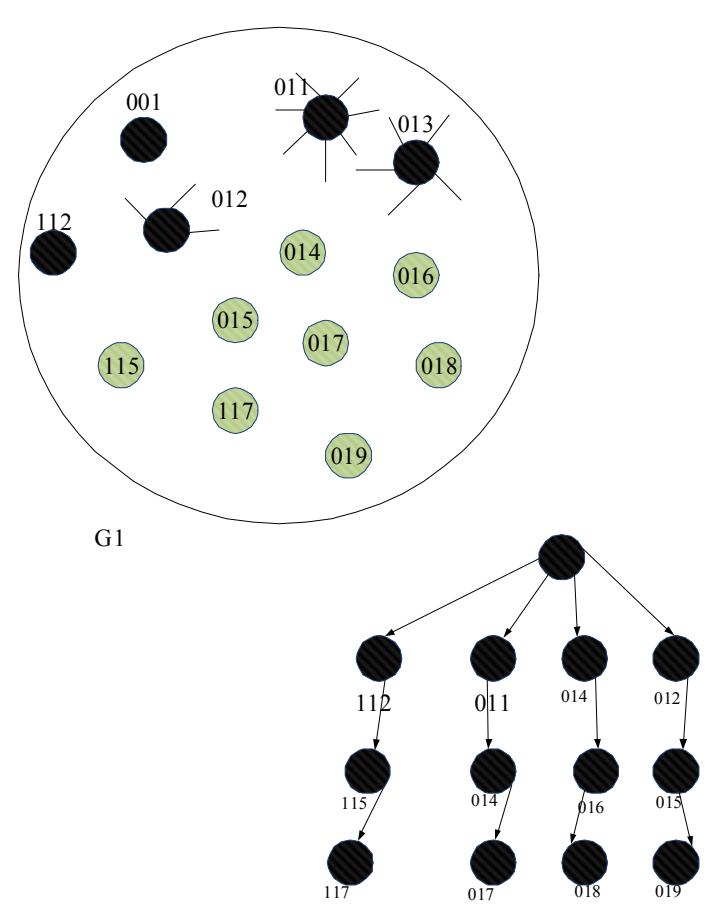

Fig. 3: Tree construction phase

heard DSM message send a response message and these responses are organized in a queue Q001. At the bottom of the figure, the tree of the leader is shown. Figure 3 represents the tree construction phase in which each discovered node $(011,012,013,112)$ has started broadcasting DSM message to discover its neighbors. At the bottom of the figure the tree that represents the cluster G1 rooted by 001 given RDST Algorithm is shown.

Power management: The idea behind our work is to increase the power saving in the cluster to increase the system life time. Feeney in ${ }^{[8]}$, has described the power consumption in transmitting (sending) in point-to-point in a Lucent IEEE 802.11 2Mbps Wave LANPCCARD, 2.4 GHZ Direct Sequence SPREAD Spectrum in a linear model as follow:

$$
\mathrm{P}_{\mathrm{T}}=\mathrm{m} * \text { size }+\mathrm{b}
$$

where, $\mathrm{P}_{\mathrm{T}}$ refers to the power consumed in sending packets, size is the size of the packet sent and $\mathrm{m}$ represents incremental cost. b represents a fixed costs. However, for sending in point -to-point $\mathrm{m}=1.9$ and $\mathrm{b}=454$. In that, the power consumed in sending $\mathrm{a}$ packet of size $\mathrm{S}$ is:

$$
\mathrm{P}_{\mathrm{T}}=1.9 * \mathrm{~S}+454
$$


$\mathrm{P}_{\mathrm{T}}$ measured by $\mu \mathrm{W}$.

Feeney shows that sending in broadcast reduces the power consumption to $\mathrm{P}_{\mathrm{T}}=1.9 * \mathrm{~S}+266$ but still dependent on the packet size.

$\mathrm{In}^{[9]}$, the distance between two nodes is determined based on, measuring the Received Signal Strength Indicator (RSSI) and based on Frii's transmission equation ${ }^{[10]}$. Given that, the remaining power at the receiving node is related quadratically to the distance between the two communicating nodes as in the following equation:

$$
\mathrm{P}_{\mathrm{r}}=\mathrm{P}_{\mathrm{T}}(\lambda / 4 \pi \mathrm{d})^{2}
$$

Where:

$\operatorname{Pr}=$ Remaining power at the receiver node

$\mathrm{P}_{\mathrm{T}}=$ Transmission power at the sender

$\lambda=$ Wave length and $\mathrm{d}$ is the distance between the two communicating nodes

So the remaining power at the receiving node is dependent on the power consumed in the transmission and on the distance. From Eq. 1 and 2 we can find that in a network using IEEE 802.11 protocol the remaining power at the receiving node is related to only the size of the packet sent and the distance between the two communicating nodes and lightly related to $\lambda$ given the following equation:

$$
\mathrm{P}_{\mathrm{r}}=(1.9 * \mathrm{~S}+454)(\lambda / 4 \pi \mathrm{d})^{2}
$$

So, we will calculate the power remaining at the receiving node taking in consideration that all the nodes in the same cluster transmit with equal $\lambda$. In that the power consumed in our network will depend on only the packet size and the distance between the two communicating node. Given that, for a fixed packet size the power remaining is dependent only on the distance d. So, instead of sending the packet at long distance the packet is sent in multihop saving small power in each steps that concludes in total power saving. For example: for sending a packet of 400 bytes between two hosts at distance $120 \mathrm{~m}$ and for $\lambda=1 / 900 \mathrm{GHz}$ and given equation 3 the power remaining at the destination node in sending in one hop (direct communication) is calculated as follow:

$$
\begin{aligned}
& \mathrm{P}_{\mathrm{rlh}}=(1.9 * \mathrm{~S}+454) \\
& (\lambda / 4 \pi \mathrm{d})^{2}=2.634 * 10^{-9} \mu \mathrm{W}
\end{aligned}
$$

However, in sending the same packet to the same destination in multihop, the power remaining at the destination will be calculated as follow:

$\mathrm{P}_{\mathrm{r}}=\operatorname{Pr}_{1}+\operatorname{Pr}_{2}$ where $\operatorname{Pr}_{1}, \operatorname{Pr}_{2}$ are the power remaining at the first hop and the second hop respectively.

$$
\begin{aligned}
& P_{r}=(1.9 \times S+454)(\lambda / 4 \pi d)^{2}+ \\
& (1.9 \times S+454)(\lambda / 4 \pi d)^{2}=2 \operatorname{Pr}_{1}
\end{aligned}
$$

Considering that all the nodes are similar, that means the power consumption in transmitting a packet is the same in all nodes, from the algorithm construction the levels in the tree are with equal distance. That means, $d$ is the same $d=120 / 2=60 \mathrm{~m}$ In that $\mathrm{P}_{\mathrm{r}}=2.1074 * 10^{-8}$.

So, the power saving in sending in multihops is:

$$
\mathrm{P}_{\mathrm{s}}=\mathrm{P}_{\mathrm{r}}-\mathrm{P}_{\mathrm{r} 1 \mathrm{~h}}=1.84 \times 10^{-8}
$$

\section{RESULTS}

The variation of power remaining with the distance and the packet size: To study the variation of power remaining with the variation of distance we have fixed the packet size and then we have varied the distance between the two communicating nodes from 120-30 m. Then, we calculate the power remaining at the destination node in each case. Table 1 shows that, the power remaining is related inversely with the distance between the two communicating nodes (by multihops). That is because reducing the distance traveled reduces the power consumption that increases the power remaining.

The variation of power consumption with the number of hops: Our goal in this work is the reduction of the power consumption in packet transmission, which means we propose a mechanism that allows the

\begin{tabular}{|c|c|c|c|c|}
\hline $\begin{array}{l}\mathrm{d}(\mathrm{m}) \rightarrow \\
\text { Size } \\
\text { (bytes) } \downarrow\end{array}$ & $\begin{array}{l}120 \\
\mathrm{P}_{\mathrm{r}} \times 10^{-10} \\
(\mu \mathrm{W})\end{array}$ & $\begin{array}{l}60 \\
\mathrm{P}_{\mathrm{r}} \times 10^{-10} \\
(\mu \mathrm{W})\end{array}$ & $\begin{array}{l}40 \\
\mathrm{P}_{\mathrm{r}} \times 10^{-10} \\
(\mu \mathrm{W})\end{array}$ & $\begin{array}{l}30 \\
\mathrm{P}_{\mathrm{r}} \times 10^{-10} \\
(\mu \mathrm{W})\end{array}$ \\
\hline 200 & 3.67 & 14.7 & 33.0 & 58.7 \\
\hline 280 & 4.34 & 17.4 & 39.1 & 69.4 \\
\hline 300 & 4.51 & 18.0 & 40.6 & 72.1 \\
\hline 350 & 4.93 & 19.7 & 44.3 & 78.8 \\
\hline 500 & 6.18 & 24.7 & 55.6 & 98.9 \\
\hline
\end{tabular}
communication by multihops to increase the system life time.

Table 2, shows that the power remaining increases significantly when the packet travels more than one hop with shorter distance.

Table 1: The variation of power remaining with the distance and the 
Table 2: The variation of power remaining with the number of hops

\begin{tabular}{lllll}
\hline $\begin{array}{l}\text { No. of Hops } \rightarrow \\
\begin{array}{l}\text { Size } \\
\text { (byte) } \downarrow\end{array}\end{array}$ & $\begin{array}{l}1 \\
\mathrm{P}_{\mathrm{r}} \times 10^{-10} \\
(\mu \mathrm{W})\end{array}$ & $\begin{array}{l}2 \\
\mathrm{P}_{\mathrm{r}} \times 10^{-10} \\
(\mu \mathrm{W})\end{array}$ & $\begin{array}{l}3 \\
\mathrm{P}_{\mathrm{r}} \times 10^{-10} \\
(\mu \mathrm{W})\end{array}$ & $\begin{array}{l}4 \\
\mathrm{P}_{\mathrm{r}} \times 10^{-10} \\
(\mu \mathrm{W})\end{array}$ \\
\hline 200 & 3.67 & 29.4 & 99.1 & 235 \\
280 & 4.34 & 34.7 & 117 & 278 \\
300 & 4.51 & 36.1 & 122 & 288 \\
350 & 4.93 & 39.4 & 133 & 315 \\
500 & 6.18 & 49.4 & 167 & 396 \\
\hline
\end{tabular}

Table 3: The variation of the power consumption and the power transmission with the packet size

\begin{tabular}{lcrrrrr}
\hline $\mathrm{d}(\mathrm{m}) \rightarrow$ & 120 & \multicolumn{1}{c}{60} & \multicolumn{1}{c}{40} \\
Size (bytes) $\downarrow$ & \multicolumn{1}{c}{$\mathrm{P}_{\mathrm{T}}$} & \multicolumn{1}{c}{$\mathrm{P}_{\mathrm{c}}$} & \multicolumn{1}{c}{$\mathrm{P}_{\mathrm{T}}$} & \multicolumn{1}{c}{$\mathrm{P}_{\mathrm{c}}$} & \multicolumn{1}{c}{$\mathrm{P}_{\mathrm{T}}$} & \multicolumn{1}{c}{$\mathrm{P}_{\mathrm{c}}$} \\
\hline 200 & 834 & 833.9 & 834 & 833.9 & 834 & 833.9 \\
300 & 1024 & 1023.9 & 1024 & 1023.9 & 1024 & 1023.9 \\
500 & 1404 & 1403.9 & 1404 & 1403.9 & 1404 & 1403.9 \\
\hline
\end{tabular}

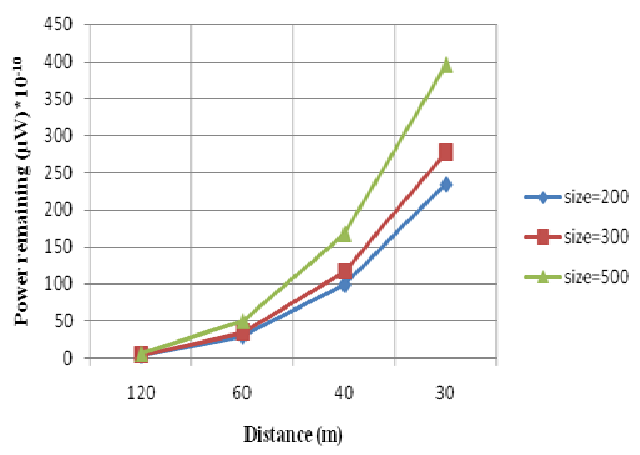

Fig. 4: The variation of power remaining with the distance

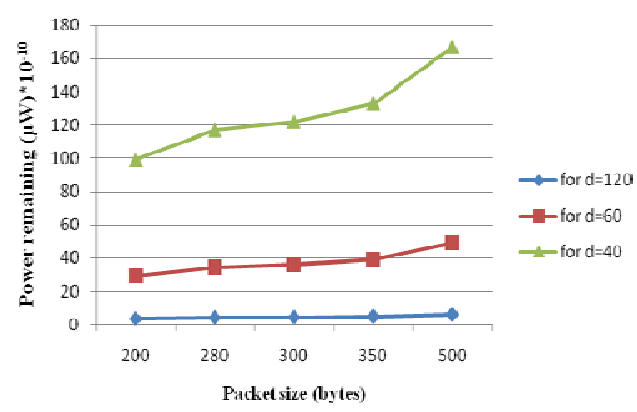

Fig. 5: The variation of power remaining with the packet size

which confirms our proposed idea that results in a total power saving and increasing in system life time.

\section{DISCUSSION}

Analyzing Eq. 3, we can note that there are only two factors that influence the power remaining at the destination node; they are the packet Size (S) and the distance between the two communicating nodes (d). In our results, we have studied the variation of power remaining and power consumption with these two factors in a cluster of 20 nodes. The data presented in Table 1 and from Fig. 4, it is evident that for a distance $120-60 \mathrm{~m}$, in all cases, the value of power remaining is equal. However, for very small distance the power remaining is increased significantly when the packet size is increased. That because the packet travels less distance and therefore, the signal dissipation will be minimized and the power consumed in this case is only that needed for the transmission. Reading Tables 1, 3 by columns we can see that the power remaining is slightly affected by the packet size.

From Table 3, the power consumption $\mathrm{P}_{\mathrm{c}}$, where the power consumption is calculated as follow: $\mathrm{P}_{c}=\mathrm{P}_{\mathrm{T}^{-}} \mathrm{P}_{\mathrm{r}}$ and power transmission $\mathrm{P}_{\mathrm{T}}$ are increased linearly with the packet size. From Fig. 5, it is clear that the power remaining varies with the packet size for a distance of $120 \mathrm{~m}$.

\section{CONCLUSION}

Clustering has become an important approach to manage MANET. In dynamic Ad-Hoc networks, it is very hard to construct an efficient network topology. By clustering the entire network the size of the problem is reduced into small sized clusters. In MANET, nodes of the same cluster must be close in order to minimize the intra-cluster communication to achieve this, the network must be divided into clusters where two member nodes of the same cluster are k-hops from each other. The algorithmic complexity of K-clustering is NP-complete problem for a simple undirected $\operatorname{graph}^{[11]}$.

In this research, we have proposed the use of local search algorithms to enhance the performance of the clustered system proposed in $^{[6]}$. We have organized the nodes of the single cluster in a tree of fixed distance levels given a determined fitness function. The communication between any node and the root in the tree is done by levels. The idea on which our study is based on the reduction of the power consumption for the communication in the single cluster by using short distance communications. Reducing the power consumption in each cluster results in a total power saving for the entire network. Our analytical results show that, in sending a packet, the power remaining at the destination node is inversely related to the distance between the two communicating nodes. Where the power remaining is increased at the destination node if the distance between the two communicating nodes is decreased. However, the power consumption is linearly increased with the packet size. Gentile et al. ${ }^{[12]}$, has proposed a distributed routing algorithm to minimize 
the overhead message for multihops routes with minimum power in MANETS. They assumed a linear model for the motion of the nodes. By applying this model, they proposed a kinetic minimum spanning tree construction. After the spanning tree is constructed, each node sets its state to cluster head. The worst case of time complexity is $\mathrm{O}\left(\mathrm{n}^{2}\right)$ where $\mathrm{n}$ is the number of nodes. The simulation results showed that stable clusters are formed. More stable cluster, means less reaffiliation process and less is the overhead imposed by the clustering mechanism. $\mathrm{LEACH}^{[13]}$, is a simple and effective distributed clustering algorithm in which the sensor nodes elect themselves as cluster head with some probability and broadcast their decisions. The remaining nodes join a cluster of which the cluster head is closest in term of the communication energy cost. The role of cluster head is periodically rotated among the nodes to balance energy consumption. Since cluster heads have the extra burden of performing large range transmission to a distant sink node. $\mathrm{In}^{[14]}$, is proposed a routing algorithm that minimizes the variance of the remaining energy of the nodes in the networks. The goal is to increase network life time. The simulation results show that the main factors that affect the performance of the routing protocols and the network life time are: the ratio of packet generated and the transmission range. Assuming a transmission range from $120-200 \mathrm{~m}$, the results confirm that increasing the transmission range, means long distance communication, will discharge the battery faster. A comparison between the proposed algorithm LRP (Life Time Prediction Routing) and DSR (Dynamic Source Routing ${ }^{[15]}$ ) is done. $\mathrm{In}^{[14]}$, the comparison results show that LPR prolong the network life time because it is based on local decisions with minimum overhead. $\operatorname{In}^{[1]}$, is proposed a new method for constructing the clusters based on the concept of k-tree core. K-tree is a tree which minimizes the sum of distances from all other nodes in the tree. The idea is to construct a spanning tree in which is determined a node called k-tree core where the distances to all other nodes in the tree is minimized. The algorithm works in two phases, in the first phase is constructed a forest, in the second phase is joined all these forest and the core k-tree is founded. The intermediate nodes in the tree send $\mathrm{k}^{-1}$ messages from its children to the parent. The root chooses the first $\mathrm{k}^{-1}$ of the leaves as the end points of the $\mathrm{k}$-tree. The root itself forms one leaf of the k-tree core. The simulation results show that increasing the value of $k$ beyond a point adds redundant edge that influences in the computation of $\mathrm{k}$-tree core. The optimal value of $\mathrm{k}$ is 5 . In addition, if the mobility is increased the number of controls packet exchanged is increased, that means the overhead is increased. $\mathrm{In}^{[2]}$, is proposed a technique based on genetic algorithm to optimize the performance of the weighted clustering algorithm in a manner to minimize the number of clusters and cluster heads. WCA (Weighted Clustering Algorithm ) ${ }^{3,4]}$, selects the cluster head based on weight of each node. The weight is calculated given the degree of the nodes, the sum of the distances of the members of the cluster head, the average speed of the nodes and the accumulative time of a node being a cluster head. The node with minimum weight is chosen to be the cluster head. The algorithm terminates once all the nodes either become a cluster head or a member of a cluster head. The idea behind the use of Genetic Algorithms (GA) is to use the mechanism of a natural selection and genetics such as reproduction, gene crossover, and mutation to find a solution of the problem of optimal selection of the cluster head that is an NP-hard problem ${ }^{[5]}$. The optimization of WCA consists on minimizing the network load and balancing the nodes among clusters. To have a smaller number of cluster heads, each cluster head must serve the maximum possible number of nodes within their clusters. By balancing the nodes among clusters, the life time of individual nodes will increase and no one node will use their battery power more than necessary. $\mathrm{In}^{[2]}$, the chromosome with lowest fitness value is chosen to be the best chromosome in that population for that generation. Each chromosome will be encoded using a string of integer that represents the node ID. At the beginning, the fitness value is equal to 0 , each node is assigned to gene, the node that is not already a cluster head and its degree is less than or equal to Max_degree is assigned to be a cluster head. The weight $\mathrm{W}$ of a node is calculated using WCA, then is inserted its ID to the cluster head set and $\mathrm{W}$ is added to the fitness value. If new child is better than the parent will be replaced with the parent. The simulation results show that after every dominant set update (cluster head, set) there is a gradual increase in LBF (Load Balanced Factor) this is due to the diffusion of the nodes among clusters. However, when the transmission range is increased from 10-35 $\mathrm{m}$ the reaffiliation (joining a new cluster) is increased. Then, for transmission range from $35-70 \mathrm{~m}$ the reaffiliation is decreased for different network size, which increases the communication overhead. WCA and its optimization have improved the performance of clustering algorithms compared to other previous clustering algorithms. But, the high mobility nodes will lead to high frequency of reaffiliation that increases the network overhead. $\operatorname{In}^{[11]}$, is proposed an Entropy Based Weighted Clustering Algorithm (EWCA) that improves the performance of WCA especially on the number of clusters and the 
reaffiliation frequency. Where it is modified two parameters are used to calculate the weights in WCA are: the average of moving speed of nodes is substituted by the entropy of local networks, which measures the disorder in a system which is a good indicator of the stability and mobility of the Ad-Hoc networks. The second parameter is that it is used the Tabu Search (TS) to optimize the cluster head election instead of genetic algorithms. Tabu Search outperforms the genetic algorithms in solving combinatorial optimization problems. The idea of TS has inspired from human intelligence procedure. TS introduce a policy to forbid certain classified moves. Attributes with good fitness value are marked in the Tabu list, to prevent cycling so that the solution space can be enlarged. The simulation results show that the effects of introducing the entropy parameters in term of the number of reaffiliation per time unit has results in decreasing the number of reaffiliation compared to the original WCA for a network of 30 nodes. The introducing of a Tabu Search has conducted to a cost decreasing with the increasing in the number of iteration compared to a fixed cost in WCA; consecutively EWCA has produced less number of clusters compared to WCA that increases the system life time.

In our proposed study, we have resolved the problem of frequently reaffiliation by organizing the nodes in a tree given the distance to the root ( head cluster ) and in ignoring the nodes with distance more than $\mathrm{r} * 80 \%$ form the root because this node has high probability to change cluster given mobility and to go out of range.

\section{REFERENCES}

1. Srivastava, S. and R.K. Ghoshi, 2002. Cluster based routing using a k-tree core backbone for mobile Ad-Hoc networks. Proceedings of the 6th International Workshop on Discrete Algorithms and Method for Mobile Computing and Communications. Sep. 28-28, Atlanta, Georgia, USA., $\quad$ pp: 72-79. http://portal.acm.org/citation.cfm?id=570820

2. Turgut, D., S.K. Das, R. Elmasri and B. Turgut, 2002. Optimizing clustering algorithm in mobile Ad-Hoc networks using genetic algorithms. In: Approach. Global Telecommunication Conference, Nov. 17-21, IEEE Xplore, USA., pp: 60-66. http://ieeexplore.ieee.org/Xplore/login.jsp?url=/iel5 /8454/26632/01188042.pdf?arnumber $=1188042$
3. Chatterjee, M., S.K. Das and D. Turgut, 2000. An on demand weighted clustering algorithm (WCA) for Ad-Hoc networks. Proceedings of the IEEE, Nov. 2000, San Francisco, pp: 1697-1701. http://citeseerx.ist.psu.edu/viewdoc/summary?DOI $=10.1 .1 .23 .185$

4. Chatterjee, M., S.K. Das and D. Turgut, 2002. WCA: A weighted clustering algorithm for mobile Ad-Hoc networks. J. Cluster Comput., 5: 193-204. http://www.ingentaconnect.com/content/klu/clus/2 002/00000005/00000002/00394386? crawler=true

5. Basagni, S., I. Chlamtac and A. Farrago, 1997. A generalized clustering algorithm for peer-to-peer networks. In: Proceeding of Workshop on Algorithmic Aspects of Communication (Satellite Workshop of ICALP), July 7-11, Bologne-Italy, http://citeseerx.ist.psu.edu/viewdoc/summary?DOI $=10.1 .1 .24 .145$

6. Zabian, A., A. Ibrahim and F. Al Kalani, 2008. Dynamic head cluster election algorithm for clustered Ad-Hoc networks. J. Comput. Sci., 4: $\quad$ 42-50. http://www.accessmylibrary.com/coms2/summary 0286-35987641_ITM

7. Russel, S. and N. Peter, 2003. Artificial Intelligence a Modern Approach. 2nd Edn., Prentice Hall, pp: 94-129. ISBN: 0-13-080302-2

8. Feeney, L.M. and M. Nillson. 2001. Investigating the energy consumption of a wireless network interfaces in ad-hoc networking environment. IEEE Infocom., $\quad 3$ : 1548-1557. DOI: 10.1109/INFCOM.2001.916651

9. Blumenthal, J., F. Reichenbach and D. Timmermann, 2006. Minimal transmission power vs.signal strength as distance estimation for localization in wireless sensor networks. Annual IEEE Communalization Society Sensor ad-hoc Commununication Networks SECON'06, pp: 761766. DOI: 10.1109/SAHCN.2006.288558.

10. Liu, C.H., D.J. Fang. Y.T. Lo and S.W. Lee, 1988. Antenna Handbook: Theory, Applications and Design. Van Nostrand Reinhold. 1st Edn., Springer, New York, pp: 1-1. ISBN-10: 0442258437.

11. Fernandess, Y. and D. Malki. 2002. K-Clustering in Wireless Ad-Hoc Networks. In Proceedings of the Second ACM International Workshop on Principles of Mobile Computing, pp: 31-37. http://DOI.acm.org/10.1145/584490.584497

12. Gentile, C, J. Haerri and R.F. Vandyck, 2002. Kinetic minimum power routing and clustering in mobile Ad-Hoc networks. Proceeding Vehicular Tech Conference, VTC 2002-Fall, pp: 1328-1333. DOI: 10.1109/VETECF 20021040431 
13. Heizelman, W., A. Chandrakasan and H. Balakrishnan, 2000. Energy-efficient communication protocol for wireless microsensor networks. Proceedings of the 33rd Hawaii International Conference on System Science, Jan. 4-7. $\quad$ pp: 10. http://ieeexplore.ieee.org/xpl/freeabs_all.jsp?arnum ber $=926982$

14. Maleki, M., K. Dantu and M. Pedram, 2003. Life time prediction routing in mobile ad-hoc networks. In: Wireless Communications and Networking Conference, Mar. 20-20, New Orland La-USA., pp: 1185-1190.

http://citeseerx.ist.psu.edu/viewdoc/summary?DOI $=10.1 .1 .60 .8203$
15. Johnson, D.B., D.A. Maltz, Y. Chun $\mathrm{Hu}$ and J.G. Jetecheva, 2001. The Dynamic Source Routing for Mobile Ad-Hoc Wireless Networks. In: Ad-Hoc Networking, Charles Perkins, E., (Ed.). Addison Wesley, pp: 139-172. 